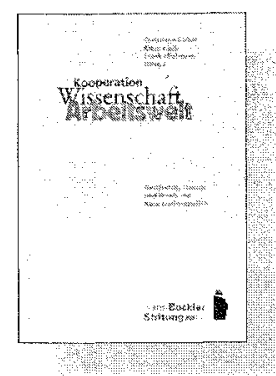

Christiane Färber/Klaus Kock/Frank Mußmann/Irmtraud Schlosser (Hrsg.)

\section{Kooperation Wissenschaft}

Arbeitswelt

Geschichte, Theorie und Praxis von Kooperationsstellen

$2003-197$ S. $-€ 24,80-$ SFR 42,90 ISBN 3-89691-543-6

Michael Jonas/Sabine Nover/ Ursula Schumm-Garling (Hrsg.)

Brennpunkt "Arbeit ${ }^{\text {ss }}$

Initiativen für eine Zukunft der Arbeit

(Schriftenreihe Hans-Böckler-Stiftung) $2002-243$ S. $-€ 24,80-$ SFR 42,90 ISBN 3-89691-528-2

Irmtraud Schlosser/

Karl Lauschke (Hrsg.)

Demokratie und Alltagshandeln

Individuelle Selbstbestimmung,

kollektive Mitbestimmung und

Solidarität

Festschrift für Bodo Zeuner

zum 60 . Geburtstag

2002 - 305 S. $-€ 29,80-$ SFR 51,00

ISBN 3-89691-536-3

\section{WESTFÄLISCHES DAMPFBOOT}

Dorotheenstr. 26a - 48145 Münster - Tel. 0251/6086080 - Fax 0251/6086020 e-mail: info@dampiboot-verlag.de · http://www.dampfboot-verlag.de

Herausforderungen in Europa

\section{Bernard Schmid}

Reiner Hoffmann (Hrsg.)

Zwischen Kontiniutät

und Modernisierung

Gewerkschaftliche

2. Aufl. $2002-435$ S. $-€ 35,00-$ SFR 62,00

A

in Zusammenarbeit mit dem EGI - Euro-

päisches Gewerkschaftsinstitut

\section{Wolfram Wassermann}

\section{osrate}

Akteure fur Demokratie in der

Arbeitswelt

2002 - 262 S. - E 24,80 - SFR 45,00 ISBN 3-89691-523-1

Jürgen Prott/Axel Keller

Hauptamtliche - Zerreißproben ortlicher Gewerkschaften

(Schriftenreihe-Hans-Böckler-Stiftung)

$2002-413$ S $-€ 24,80-$ SFR 45,00

ISBN 3-89691-401-4

Wolf Jürgen Röder

Klaus Dörre (Hrsg.)

(B)

im flexiblen

\section{Kapitalismus}

$-2085-€ 19,80-$ SFR 34,50

SBN 3-89691-535-5 der Regel nicht. gewerkschaftlichen Pluralismus existieren.

\title{
SUD und Solidaires: Linksalternative Basisgewerkschaften in Frankreich
}

Wer in den letzten Jahren an einer Demonstration in Frankreich oder unter Beteiligung von Franzosen und Französinnen teilgenommen hat, etwa aus An lass eines internationalen Gipfels der Staats- und Regierungschefs von Europäischer Union oder G8-Gruppe, der dürfte sie wahrgenommen haben, die in vielen Farben auftauchenden Fahnen mit der Aufschrift SUD. In neuerer Zeit ist der Schriftzug meist zu SUD-Solidaires ergänzt worden. Auch bei größeren gesellschaftlichen Konflikten wie anlässlich der massiven Erwerbslosenproteste im Winter 1997/98 oder, in jüngerer Zeit, dem über vier Monate andauernden Streik der Beschäftigten einer Pariser McDonalds-Filiale fehlen die Symbole dieser relativ kleinen, aber höchst aktiven Gewerkschaftsorganisation in

1. Der historische und juristische Kontext: Ein System des

Dass neue Akteure wie die SUD-Gewerkschaften im Raum eines guten Jahrzehnts einen veritablen Durchbruch in vielen Sektoren schaffen konnten, ist neben anderen Ursachen - auch mit auf den Kontext zurückzuführen, in dem sich die französische Gewerkschaftsbewegung organisiert. Dieser Kontext ist seit Jahrzehnten von einer Tradition des politisch-ideologischen Pluralismus geprägt: Mehrere Gewerkschaften unterschiedlicher Orientierung können, prinzipiell auch im selben Betrieb oder in derselben Branche, nebeneinander

Diese plurale Zusammensetzung der französischen Gewerkschaftsbewegung führte schon früh zu rechtlichen Problemen: Wer sollte befugt sein, für die abhängig Beschäftigten zu sprechen und ein für alle Lohnabhängigen in einem Betrieb oder einer Branche verbindliches Kollektivabkommen (das französische Äquivalent zum deutschen Tarifabkommen) abzuschließen? Das geltende französische Recht organisiert eine „Repräsentation in der Pluralität “. Jede einzelne der verschiedenen, miteinander konkurrierenden Gewerkschaften ist berechtigt eine convention collective zu unterzeichnen, die rechtsverbindliche Wirkung (ggf. bis zum nächsten Konflikt) entfaltet - aber unter einer 
Voraussetzung. Dabei dreht sich alles um den zentralen Begriff der représentativité, also des „repräsentativen Charakters ${ }^{66}$ einer Gewerkschaftsorganisation. Wenn eine Gewerkschaft in diesem Sinne „repräsentativ" ist, kann sie einen für alle Beschäftigten bindenden Kollektivvertrag unterzeichnen.

Bis in die jüngere Vergangenheit hinein hatte diese rechtliche Konzeption kaum problematische Implikationen: Erstens aufgrund des im französischen Arbeitsrecht geltenden Günstigkeitsprinzips - der Artikel L. 132-4 des Arbeitsgesetzbuchs schreibt im Prinzip vor, dass das Kollektivabkommen oder die Betriebsvereinbarung nur solche Bestimmungen enthalten darf, die aus Sicht der abhängig Beschäftigten günstiger ausfallen als jene der geltenden Gesetzgebung. Dennoch wird diese Ermächtigung einer - möglicherweise in deutlicher Minderheitsposition befindlichen - einzelnen Gewerkschaft, ein für alle Beschäftigten verbindliches Abkommen zu schließen, in jüngerer Zeit immer mehr zum Problem. Denn einerseits hat der Gesetzgeber seit 1982 - die erste Bresche in das Günstigkeitsprinzip hat ironischerweise die erste Linksregierung unter François Mitterrand gerissen - den Verhandlungspartnern die Möglichkeit eröffnet, auf bestimmten Feldern Abkommen (die so genannten accords dérogatoires) zu schließen, deren Bestimmungen von der geltenden Gesetzgebung abweichen, ohne (notwendigerweise) günstiger aus Sicht der Lohnabhängigen zu sein. Andererseits hat auch die (richterliche) Auslegung des Günstigkeitsprinzips zu einer gewissen Erosion beigetragen. Hat doch eine viel beachtete höchstrichterliche Entscheidung des Obersten Gerichtshofs vom 19. Februar 1997 in einem konkreten Fall anerkannt, dass eine Betriebsvereinbarung auch dann als „günstiger" im Vergleich zu konkurrierenden Regeln gelten konnte, wenn die Lohnabhängigen „Opfer" in Form des Verzichts auf einen Lohnzuschlag zu bringen hatten, aber diese „Opfer" durch den Erhalt der Arbeitsplätze gerechtfertigt wurden. Allerdings ist die in diesem Streitfall angewendete Lösung in anderen Fällen durch die Gerichte abgelehnt worden.

Ein zweiter wichtiger Faktor, der den ,gewerkschaftlichen Pluralismus“ akzeptierbar macht, besteht in der in Frankreich vorherrschenden Konzeption des Streikrechts. Dieses durch die Verfassung garantierte Grundrecht stellt - anders als in Deutschland - kein "organisches“ Recht dar, dessen Ausübung an die gewerkschaftliche Struktur gebunden ist. Vielmehr gilt das Streikrecht als individuelles Grund- und Freiheitsrecht jedes abhängig Beschäftigten, das die-

1 Schließen mehrere „, repräsentative“ Gewerkschaften einen Kollektivvertrag bzw. eine Betriebsvereinbarung mit dem Arbeitgeber oder Kapitalverband, so sind alle diese Abkommen rechtsgültig, sofern sie verschiedene Themen betreffen. Haben sie aber den gleichen Gegenstand und sind sie auf gleicher Ebene abgeschlossen, so gilt das jeweils jüngere Ablommen als "Revision“ des jeweils älteren. Dazu stellt der Artikel L. 132-7 Arbeitsgesetzbuchs eigene Regeln auf. Nach diesen Bestimmungen kann etwa, unter restriktiv gefassten Voraussetzungen, eine af. Nacher äleren Abommens gegen die Revision" während der ersten Tage ein Veto einlegen. ser auch völlig unabhängig von einer gewerkschaftlichen Struktur ausüben kann - jedenfalls unter einer Bedingung: Das Streikrecht „in konzertierter Form" auszuüben, also durch mehr als einen einzigen Beschäftigten (nämlich mindestens zwei). Diese Konzeption macht die abhängig Beschäftigten weniger abhängig von der Existenz und der konkreten Politik einer gewerkschaftlichen Struktur. Sie hat zugleich die Entstehung freier (Streik-)Koordinationen, namentlich in den Jahren 1986 bis 1989, außerhalb gewerkschaftlicher Apparate begünstigt. Aus diesen coordinations wiederum ist, $\mathrm{zu}$ maßgeblichen Teilen, der Kern der ersten SUD-Gewerkschaften entstanden.

\section{Auswirkungen auf die Gründung der SUD}

Der Gesetzgeber hat eine Reihe von Rechtspositionen und Vollmachten auf die „repräsentativen“ Gewerkschaften beschränkt: Diese können rechtsverbindliche Kollektivabkommen und Betriebsabkommen abschließen. Sie können ab einer bestimmten Betriebsgröße einen (ganz oder teilweise) Freigestellten benennen, einen délégué syndical. Und sie können, seit einem Gesetz von 1982, als einzige im ersten Wahlgang der Wahlen zu Personalvertretungen (etwa zu Betriebsräten oder für die délégues du personnel, die eine Art betrieblicher Vertrauensleute darstellen) antreten. Ein zweiter Wahlgang, zu dem dann alle Gewerkschaften - auch die nicht als syndicats représentatifs anerkannten - antreten dürfen, findet nur dann statt, wenn die Wahlbeteiligung im ersten Durchgang unter 50 Prozent lag. Daneben verwalten die „repräsentativen" Gewerkschaften gemeinsam mit Vertretern der Arbeitgeber eine Reihe von Sozialkassen des öffentlichen Sozialversicherungssystems.

Die Beschränkung dieser Rechtsansprüche auf die „repräsentativen“ Gewerkschaften geht auf ein Gesetz vom 28. Mai 1945 zurück. Damals ging es vorrangig darum, die Reste der vom profaschistischen Vichy-Regime ererbten, korporatistischen Vereinigungen außen vor zu halten. Doch in den darauf folgenden Jahren, nach Ausbruch des Kalten Krieges, trieb eine andere Frage Regierungen und Arbeitgeberlager um: Wie konnte man das Gewicht des mit Abstand stärksten Gewekschaftsbunds umgehen, der "pro-kommunistischen" CGT, und trotzdem über legitim erscheinende Verhandlungspartner verfügen? Ein Gesetz vom 11. Februar 1950 legt seitdem die Kriterien für „Repräsentativität" fest. Diese sind im Artikel L. 133-2 des Arbeitsgesetzbuchs niedergelegt, der fünf Kriterien nennt: die Mitgliederzahl, die Unabhängigkeit (gemeint ist jene gegenüber dem Arbeitgeber), die Mitgliedsbeiträge, die „Erfahrung und Lebensdauer der Gewerkschaft“, sowie „die patriotische Haltung während der Besatzung ". Allerdings brauchen jene Gewerkschaften, die in einem der fünf "groß̧en" Dachverbände (CGT, CFDT, FO, CFTC und CGC) - von denen zumindest zwei, die christliche CFTC und die Gewerkschaft der höheren Angestellten CGC, in Wirklichkeit eher geringen Einfluss haben - Mitglied sind, 
ihre „Repräsentativität" nicht erst zu beweisen, sondern gelten automatisch, kraft einer „unwiderlegbaren Vermutung“, als repräsentativ. Damit werden mitunter vom Arbeitgeber aufgebaute Pseudo-Organisationen vor allem in kleineren Betrieben, die kaum Einfluss haben, aber in einen der Dachverbände Aufnahme finden, künstlich als „repräsentativ“" anerkannt.

Die Gerichte haben die Anwendung dieser Kriterien im konkreten Falle auszulegen. Dabei haben sie schon seit längerem anerkannt, dass nicht unbedingt alle dieser Punkte die gleiche Aussagekraft aufweisen. Die Richter dürfen daher einigen Kriterien stärkeres Gewicht als den anderen zusprechen, aber unter der Bedingung, nicht den Gesamteindruck unter Würdigung des Zusammenspiels aller bestehenden Kriterien zu vernachlässigen.

Besonders die SUD-Gewerkschaften hatten überall, wo sie (zum ersten Mal) auftraten, mit dem Problem der Anerkennung ihres „repräsentativen Charakters“ zu kämpfen. In der Regel führten sowohl die Betriebsleitung als auch manche der traditionellen Gewerkschaftsbünde Prozesse gegen den Neuankömmling auf der gewerkschaftlichen Bühne, um etwa sein Antreten zu Personalrats- (im öffentlichen Dienst) oder Betriebsratswahlen zu verhindern. ${ }^{2}$

Laut Annick Coupé, Mitgründerin der ersten Branchengewerkschaft SUD-PTT (SUD bei Post und Telekom) und seit 2001 Sprecherin des umgebildeten Dachverbands Union syndicale-Solidaires, wurden, in 99 Prozent der Fälle entsprechende Verfahren angestrengt, mit dem Ziel, SUD ihre „Repräsentativität $^{6 \text { z }}$ zu verweigern. Allerdings hätten die SUD-Gewerkschaftsgründer „über die Hälfte “ der Prozesse gewonnen. Freilich hätten die Prozesse oft zur Abschreckung und Entmutigung (potenzieller) Aktivisten beigetragen. Andererseits äußert der Unternehmensberater (und frühere CFDT-Gewerkschafter) Daniel Labbé in seinem Buch - das eher daraufhin konzipiert ist, Gegnern von SUD Strategien und Analyseinstrument an die Hand zu geben - die gegenläufige Ansicht: Die „Strategie der institutionellen Ausgrenzung“ etwa bei der Post habe erheblich zur Stärkung der Positionen von SUD-PTT beigetragen. Denn diese, in seinen Augen gescheiterte, Strategie habe SUD in den Augen vieler abhängig Beschäftigter als „Verteidiger der Rechtlosen“ erscheinen lassen und ihr die Aura moralischer Integrität verliehen. (Labbé 2001, 67) Aus ähnlichen Gründen scheint die Ausgrenzungsstrategie bei einigen Unternehmen, etwa

2 In den öffentlichen Diensten, wo die SUD-Gewerkschaften zuerst auftraten - bei Post und Telekom, in den Krankenhäusern - galt bis vor wenigen Jahren die Regel des Artikel L. 133-2 in dieser Form nicht, da das Arbeitsgesetzbuch unmittelbar nur auf die Unternehmen des priva ten Wirtschaftssektors (sowie die Eisenbahner mit ihrem besonderen Statut) Anwendung findet, während im öffentlichen Dienst staatlich gesetzte Sonderregeln gelten.Freilich stellten die in ihrer Rechtsform autonomen öffentlichen Unternehmen oftmals eigene „RepräsentativätsRegeln" für die bei ihnen vertretenen Gewerkschaften auf. Doch im Jahr 1996 schrieb ein Gesetz des damaligen konservativen Ministers für den öffentlichen Dienst (und jetzigen Justizministers), die Loi Perben, auch für den öffentlichen Dienst nunmehr gleiche Regeln bezüglich der gewerkschaftlichen représentativité vor.
France Télécom, ad acta gelegt worden zu sein.

Zum Jahresende 2002 erzielte SUD einen doppelten gerichtlichen Sieg. In einer Entscheidung vom 3. Dezember 2002 fand sich der Oberste Gerichtshof dazu bereit, die Anwendung der Kriterien der Repräsentativität ein wenig „aufzuweichen“: Die erwiesene Unabhängigkeit gegenüber dem Arbeitgeber die häufig bereits durch dessen Prozessieren erbracht ist - und der "Einfluss", verstanden als Fähigkeit zur Mobilisierung eines Teils der abhängig Beschäftigten, können demnach für den Nachweis der „Repräsentativität" genügen. Konkret hatten die Betriebsleitung und die CFDT gegen die im Januar 2001 neu gegründete Gewerkschaft SUD bei den elsässischen Sparkassen geklagt, nachdem SUD drei Monate später einen Freigestellten ernannt hatte (vgl. dazu die Analyse der Wirtschaftszeitung La Tribune vom 5.12.2002). Wenige Tage später gab, am 16. Dezember 2002, das Pariser Verwaltungsgericht einer Klage der - im Aufbau befindlichen - Gewerkschaft SUD Transports statt. Diese hatte die Personalratswahlen bei den Pariser Transportbetrieben der RATP (Metro- und Buslinien) angefochten, da SUD nicht am ersten Wahlgang hatte teilnehmen können (Le Monde vom 19.12.2002).

Nicht unwahrscheinlich ist unterdessen, dass die neokonservative Regierung Raffarin in den ersten Monaten des Jahres 2003 ohnehin die geltenden Regeln in Sachen "Repräsentativität" in Frage stellen wird. Denn auch im Arbeitgeberlager werden diese Spielregeln zunehmend in Frage gestellt, weil ihre Anwendung sich als untauglich erweist, um „sozialen Frieden" bzw. Ruhe bei den Arbeitskonflikten herzustellen. Denn oftmals weisen die auf solcher Grundlage geschlossenen Abkommen nur eine geringe Legitimation auf, und können in der Praxis schon bald durch einen neuen Konflikt über den Haufen geworfen werden. Daher verlangt auch ein Teil der Arbeitgeber nach einer Neuregelung, die zu stärkerer Legitimierung der unter „Sozialpartnern“ geschlossenen Vereinbarungen führen soll. Die SUD-Gewerkschaften wollen die sich dadurch eventuell öffnende Situation nutzen, um die Frage nach der gewerkschaftlichen Demokratie öffentlich neu aufzuwerfen.

\section{Der Hintergrund der Entstehung von SUD:}

Die widersprüchliche Entwicklung der CFDT 1968 - 1988

Die Wurzeln der Entstehung des linksalternativen Gewerkschaftsprojekts SUD finden sich in jener widersprüchlichen Entwicklung, welche die CFDT - neben der CGT der zweite große gewerkschaftliche Dachverband - ab Mitte der 60er Jahre nahm. Eine der damals in der CFDT (neu) angelegten Traditionslinien wird später gewissermaßen durch die SUD-Gewerkschaften verlängert, nachdem die CFDT sie in einem längeren Prozess abgestoßen hatte.

Die Confédération française démocratique du travail (Französischer demokratischer Arbeiterverband) entstand durch eine Umwandlung des Mehrheits- 
blocks der alten, katholisch-sozialen Konföderation CFTC anlässlich ihres Kongresses vom 6./7. November 1964 in Issy-les-Moulineaux. Im Zuge der Modernisierung der französischen Nachkriegsgesellschaft sprach sich der Kongress für eine „Entkonfessionalisierung ${ }^{66}$ des Gewerkschafts- und Gesellschaftsverständnisses aus. Eine eher konservativ geprägte Minderheit wollte den Wandlungsprozess jedoch nicht mittragen und spaltete sich ab, um den $\mathrm{Na}$ men der CFTC beizubehalten.

Die neu entstandene CFDT nahm ab Mitte der 60er Jahre an bedeutenden Streikbewegungen teil. Zugleich fand auch eine Öffnung zu neuen gewerkschaftlichen Themen statt, wie beispielsweise „Dritte-Welt ${ }^{\text {" }}$-Solidarität, Forderungen nach Lebensqualität und weniger Autoritarismus in der Gesellschaft sowie - $\mathrm{ab}$ den frühen 70er Jahren bereits - Ökologie; so forderte die CFD'T damals einen mehrjährigen Baustopp für Atomanlagen. Die ideelle Basis für diese Weiterentwicklung der CFDT-Programmatik beruhte zu bedeutenden Teilen auf einem moralisch-ethischen Anspruch, der aus dem ursprünglich christlichen Kontext herstammte, aber aus der religiösen Bindung herausgelöst wurde. Die Abgrenzung von der CGT erfolgte (abgesehen davon, dass die CFDT sich nicht gleichermaßen positiv auf den sowjetischen 'Realsozialismus' bezog) dadurch, dass die "entkonfessionalisierte "CFDT der CGT vorwarf, ein vor allem „quantitatives" Gewerkschaftsverständnis zu vertreten - im Sinne von Lohn- und Prozentforderungen, welche das gesellschaftliche "Sein“ nicht in seinem Wesen träfen. Der ursprüngliche Moralismus verband sich dabei mit einem sozialutopischen Moment, das im Laufe der 70er Jahre zeitweise stark antiautoritäre Züge annahm.

Während des Mai 1968 - der in Frankreich auch den mehrwöchigen Streik von 8 Millionen Beschäftigten umfasste - unterstützte die CFDT deutlich die Streikenden. Damit versuchte sie auch gegenüber der CGT Terrain zu gewinnen, denn die Apparate von CGT und Französischer Kommunistischer Partei (PCF), die durch den Ausbruch der Streikwelle überrascht wurden, suchten diese in erster Linie unter Kontrolle zu bringen und zu bremsen. In der Folgezeit gewann die CFDT umso leichter jüngere Neumitglieder, auch aus der „Neuen Linken“. Der CFDT-Kongress vom 5.-10. Mai 1970 beschloß, dass der Gewerkschaftsbund sich nunmehr zum „Sozialismus" als gesellschaftlichem Projekt bekennt. Damit ist vor allem der socialisme autogestionnaire gemeint, der "Selbstverwaltungs-Sozialismus“. Die autogestion ist in jenen Jahren in vieler Leute Munde, vor allem bei dem ein Jahr lang währenden Kampf um die Uhrenfabrik Lip (1973/74), die - nachdem ihre Besitzer sie in Konkurs gehen lassen wollten - durch die Arbeiter übernommen und in Eigenregie betrieben wurde.

"Selbstverwaltung" war zwar zunächst eher ein Slogan denn ein fertiges Gesellschaftsmodell, doch ließ sich durch ihn vor allem der Unterschied zur autoritären Planwirtschaft nach sowjetischem Muster hervorheben. Damit wurde auch der Unterschied zur PCF und zur CGT, als französischen Unterstützern des sowjetischen Systems, deutlich. Die CFDT machte sich die - insgesamt noch eher schwammige - Formel der autogestion zu eigen, wobei der Politologe René Mouriaux darauf hinweist, dass sich ein bestimmtes Verständnis von „Selbstverwaltung“ durchaus mit Vorstellungen eher konservativer bzw. christlicher Herkunft zum Subsidiaritätsprinzip verbinden lässt. Tatsächlich weist das katholisch-bürgerliche Milieu in Frankreich eine lange Tradition der Verteidigung „kleinerer, historisch gewachsener Einheiten“ gegenüber dem modernen, republikanischen Staat mit seinem abstrakten Gleichheitsversprechen auf (Mouriaux 1984, 106). Allerdings lehnt es der CFDT-Apparat ab, den Selbstverwaltungsanspruch auf die Marx'sche Theorie zu gründen. ${ }^{3}$

Anfang der 70er Jahre platzierte sich die Führung der CFDT an der Seite der französischen Sozialdemokratie, die ab 1971 neben der KP zur zweiten Linkspartei aufzusteigen begann. Im Jahr 1974 beteiligte sich die CFDT an den so genannten Assises du socialisme, einem Kongress, der einer deuxième gauche - der „zweiten Linken“" neben der KP - Schwung verschaffen soll (vgl. dazu Hamon/Rotman, 1982) Der CFDT-Apparat setzt damals vor allem auf Michel Rocard, der im selben Jahr 1974 von der linkssozialistischen PSU zur größeren Parti Socialiste (PS) seines langjährigen Rivalen François Mitterrand überwechselte. Viele CFDT-Kader begleiten die Rocardianer in den PS. Rocard wird später die ewige Nummer Zwei hinter dem verhassten François Mitterrand bleiben, und als Premierminister einer sozialliberalen Koalition in den Jahren 1988 bis 1991, unter Präsident Mitterrand, enden. Bis heute halten Intellektuelle aus der Umgebung von Michel Rocard höchste Führungspositionen im CFDT-Apparat inne.

So wie die CGT die Französische Kommunistische Partei begleitete, so schien die CFDT nunmehr die neuen Aufschwung nehmende Sozialdemokratie zu begleiten - die in der Union de la gauche mit der KP verbündet ist. Die Linksunion schien unaufhaltsam an die Regierung zu drängen, da das neue Gewicht der Sozialdemokratie innerhalb der Union geeignet schien, vielen Wählern die Kommunismus-Angst zu nehmen.

Doch die Linksunion zerbrach im September 1977. Einerseits hatte sich die PS, unter dem Einfluss der Sozialistischen Internationale und vor allem der westdeutschen SPD, deutlich nach rechts entwickelt. Andererseits hatte die sowjetische KP Druck auf die PCF ausgeübt. Seitens der KPdSU war man ei-

3 Später hat die CFDT, im Zuge ihrer Rechtsentwicklung, der Selbstverwaltung mit dem Slogan von der „Autonomie der Zivilgesellschaft“" erneut eine völlig veränderte Bedeutung verliehen - jetzt geht es um die Idee einer autonomen Regelungssphäre der Sozialpartner, aus der die politische und gesetzliche Regulierung weitgehend zu verdrängen sei In der tonkreten Ausformung lief dies in späten 90er Jahren vor allem darauf hinaus, sozialpolitishenkreten Axsform derlichen „Entpolitisierung" der sozialen Beziehungen, abzusegnen. 
nerseits nicht gewillt, den Einfluss auf die französische „Bruderpartei ${ }^{\text {‘s }}$ zu verlieren. Andererseits misstraute man Mitterrand auch aus außenpolitischen Gründen - weil man ihn für einen entschiedeneren "Atlantiker" hielt als die Chefs der gaullistischen Rechten.

Der Bruch der Linksunion führte zu unterschiedlichen Konsequenzen. Seitens der PCF und der CGT etwa verfiel der „harte Kern“ in eine zunchmende dogmatische Erstarrung, und beginnende politische Isolierung. Gleichzeitig gelang der CGT die von ihr erhoffte Remobilisierung der Arbeiterschaft nicht. Denn durch den ab 1974 beginnenden Anstieg der Massenarbeitslosigkeit verschlechterte sich das Kräfteverhältnis in den Betrieben drastisch. Die CGT geriet so immer stärker ins Schlepptau ihrer politischen „Mutterpartei“ (Ross 1984, besonders 71/72).

Seitens des CFDT-Apparats wiederum setzte zu jener Zeit das ein, was man als zynische Verbitterung gegenüber „der Politik“ bezeichnen kann. Da das Politikmodell der Führung gescheitert war - Michel Rocard hatte sich innerhalb des PS nicht durchsetzen können, und eine Regierungsübernahme der Linksparteien schien weit entfernt $z u$ sein - verabschiedete sich die Führung von gesamtgesellschaftlichen Visionen und sozialen Utopien. Nur noch die „eigentliche Aufgabe“ der Gewerkschaft habe ab jetzt zu zählen, nämlich das Verhandeln mit den Arbeitgebern auf der betrieblichen Mikroebene, zum Zwecke des Abschlusses konkreter Kollektivabkommen. Der CFDT-Kongress von Brest 1979 steht im Zeichen des recentrage - „rezentrieren“, der Ausdruck stammt von Generalsekretär Edmond Maire, bedeutet hier so viel wie „sich auf seine eigentlichen Aufgaben besinnen". Radikaleren gesellschaftspolitischen Ideen wird jetzt der Kampf angesagt.

Ein neuer scharfer Kurswechsel erfolgte für kurze Zeit im Frühjahr 1981. Vom CFDT-Apparat eher unerwartet, gewann der Sozialist François Mitterrand doch noch die Präsidentschaftswahl vom 10. Mai 1981. Die Linksunion war an der Regierung, wobei die KP bereits tendenziell marginalisiert war und sich völlig im Schlepptau Mitterrands befand. Zahlreiche CFDT-Führungskräfte traten in die neu gebildete Regierung von Pierre Mauroy oder in die höhere Verwaltung ein. Die CFDT übte dabei eher eine sozialpolitische Bremserfunktion aus. Edmond Maire wird zum 20. Jahrestags des Wahlsiegs Mitterrands erzählen: „Am Abend des 10. Mai warnten wir ihn (Mitterrand) vor einer 'Politik der sozialen Großzügigkeit', welche die Inflation angeheizt hätte. (...) Wir haben Mitterrand erläutert, dass man die 35-Stunden-Woche nicht sofort und nicht auf gesetzlichem Wege einführen dürfe, sondern nach und nach sowie auf dem Verhandlungsweg." (Libération, 8.5.2001) Die CFDT wird sich in der ersten Zeit gegenüber der Mauroy-Regierung verhalten, „als wäre sie eine offzielle Gewerkschaft" - so steht es im Rechenschaftsbericht eines CFDTFührungsmitglieds von Oktober 1981 (Mouriaux 1984, 93).

Als die Linksregierung ab dem Jahreswechsel 1983/84 - nach anfänglichen so- zialen Reformen - das Ruder jäh herumwirft, da der wirtschaftliche Druck auf sie rapide wächst, spielt die CFDT die Avantgarde der regressiven Bewegung. Am Amtssitz des Premierministers ruft Edmond Maire vor Journalisten dazu auf, eine notwendige „neue Austeritätspolitik“ zu betreiben und eine "Reformpause" einzulegen (Aparicio/Pernet/Torquéo, 40). Jeder Bezug auf den Sozialismus wird aus dem CFDT-Gesellschaftsprojekt gestrichen, der Markt und der Aufbau der Europäischen Gemeinschaft (sowie die „vernünftige“ Verhandlung unter Sozialpartnem) werden positiv besetzt. Soziale Bewegungen hingegen werden nunmehr, im Kontext des verallgemeinerten Zynismus gegenüber allem "Politischen ${ }^{\text {" }}$ - nur noch denunziert, wie etwa die Studierendenbewegung vom Dezember 1986 (als „Manipulation der radikalen Linken ${ }^{6}$ ). Hier schlägt die Geburtsstunde der SUD-Gewerkschaften.

\section{SUD: ein (erfolgreiches) gewerkschaftliches Experiment}

Die Entstehung bzw. das Wachstum der SUD-Gewerkschaften lässt sich in drei zeitliche Abschnitte einteilen. Am Anfang steht die Herausbildung der ersten Organisationen mit diesem Namen als Folge der hefigen inneren Konflikte in einigen CFDT-Sektionen in den Jahren 1988/89, gefolgt von einer allmählichen Konsolidierung dieser organisatorischen Kerne. Danach folgt eine Phase, die von den unmittelbaren Nachwirkungen des Streikherbstes in den öffentlichen Diensten Frankreichs im November/Dezember 1995 geprägt ist. Drittens erhalten die SUD-Gewerkschaften neue ,Zufuhr" durch die innergewerkschaftlichen Konflikte, die - dieses Mal in der Privatindustrie - aus den Effekten der "Aubry-Reform" zur Arbeitszeitpolitik erwachsen. Damit einher geht eine Verbreiterung der sozialen Basis der SUD-Gewerkschaften, die nunmehr auch in die privaten Wirtschaftssektoren hineinreicht, nachdem die „Pioniergruppen“ alle im öffentlichen Dienst entstanden waren.

Im Herbst 1988 fanden zwei größere Arbeitskämpfe statt, welche die Widersprüche innerhalb der CFDT zum Tanzen bringen. Einerseits traten die Sortierzentren der Post sowie, im Großraum Paris, die camions jaunes - die ,gelben Lastwagen" - in den Streik, gegen eine drohende Privatisierung von Teilen des Sektors und wegen der vom Arbeitgeber festgelegten, variablen Arbeitszeiten. Die dort beschäftigten Arbeitskräfte sind - anders als der Durchschnitt der Postbeschäftigten - zum größeren Teil nicht gewerkschaftlich organisiert, wofür es mehrere Gründe gibt: Sie sind oft gering qualifiziert, und viele von ihnen sind (gesellschaftlich mitunter schlecht integrierte) Zuwanderer aus den départements d'outre-mer, aus den französischen „Überseebezirken", und vor allem von den Antillen. Deswegen erfolgte der Zusammenschluss in einer Streikkoordination, in der Mitglieder und Nichtmitglieder gewerkschaftlicher Organisationen zusammen wirken.

Die CFDT-Sektion bei den PTT (Poste, télégraphes et télécommunications), 
unter deren Dach damals noch die jetzigen Post- und Telekom-Unternehmen zusammengeschlossen waren, solidarisierte sich nicht mit der Streikbewegung. Erstens richtete sich diese gegen die Regierung von Michel Rocard, und damit einen besonderen Freund der CFDT-Führung. Und zweitens befanden sich damals bereits die Pläne für die Réforme Quilès in den Schubladen, die schließlich 1991 in Kraft getretene Reform von Postminister Paul Quilès, durch welche die vormalige öffentliche Verwaltung der PTT in zwei autonome Unternehmen La Poste und France Télécom aufgetrennt wird. Dic CFDT will bezüglich der Reform konsultiert werden. So ruft der Vorsitzende der CFDTSektion PTT die Regierung am 13. November zu hartem Durchgreifen gegen die Streikenden auf. Der innergewerkschaftliche Streit zeigte seine Auswirkungen kurz darauf auf dem CFDT-Kongress, der vom 21.-28. November 1988 in Straßburg stattfand. Gewerkschaftschef Edmond Maire forderte dort explizit „,den Ausschluss der schwarzen Schafe der Unruhestiftung, von denen einige missbräuchlich den Namen der CFDT in Anspruch nehmen" (Coupé 2002, 16/17; Crettiez/Sommier 2002, 174/175; Labbé 2001, 52/52; Interview mit Guy Freyche). Die Mitgliedschaft der gesamten Sektion der CFDT-PTT im Großraum Paris im Dachverband wird „suspendiert".

Ungefähr zeitgleich begann die Streikbewegung im Gesundheitswesen, vor allem im Großraum Paris. Die Krankenschwestern und andere Gruppen von Beschäftigten leiden schon seit längerem unter der mangelnden Anerkennung ihrer beruflichen Qualifikation, den extremen Hierarchiebeziehungen gegenüber dem ärztlichen Personal und den von Stress und Hektik geprägten Arbeitsbedingungen. Auch diese Berufsgruppe ist in vergleichsweise geringem Maße gewerkschaftlich organisiert. Auch hier bildete sich rasch eine Streikkoordination. Noch während sich der mehrwöchige Streik ausweitete, unterzeichnete die CFDT-Sektion im Gesundheitswesen (CFDT Santé) ein Abkommen, das von vielen Streikenden als Dolchstoß in den Rücken erlebt wurde. Im März 1989 wurde im Anschluss die gesamte CFDT-Sektion des Großraums Paris (CFDT Santé Ile-de-France), die als erste das Abkommen öffentlich kritisiert hatte, kollektiv aus der Branchenorganisation der CFDT ausgeschlossen (Coupé 2002, 17 und 26).

Die solchermaßen an den Rand gedrängten oder ausgeschlossenen CFDTMitglieder beschlossen, ihren Kampf nicht einfach aufzugeben. Die „suspendierten" oder ausgeschlossenen Gewerkschafter bei Post und Telekom forderten die CFDT-PTT zunächst auf, alle Sanktionen bis zum 15. Januar 1989 zurückzunehmen. Zugleich hinterlegten sie bei den zuständigen Registrierungsbehörden bereits die Statuten für eine neue unabhängige Branchengewerkschaft - „für den Fall, wo dies notwendig würde“. Als Namen gaben sie Solidaires, Unitaires, Démocratiques an (für "Solidarisch, der Kampfes-Einheit verbunden und demokratisch" wobei alle Adjektive im Plural stehen). Diese Namensgebung impliziert eine klare Kritik an den Praktiken beim Dachver- band CFDT, die man nicht zu wiederholen wünscht: Die CFDT war nicht solidarisch gegenüber den Streikenden, und hat auf undemokratischem, autoritärem Wege die Aktivitäten ihrer eigenen Mitglieder zu unterbinden versucht. Als Abkürzung ergibt dies SUD (gleichklanglich mit "Süden"), ein Name, der zugleich ein Augenzwinkern für die zahlreichen bei der Post arbeitenden „Provinzler ${ }^{6 s}$ aus Südfrankreich darstellen, und an Sonnenschein und andere angenehme Dinge denken lassen soll. Am 1. Februar 1989 war die Gründung dann offiziell. In den Krankenhäusern, wo der Konflikt mit der CFDT ein wenig zeitverschoben stattfand, wurde als Name CRC gewählt. Das Kürzel steht für Coordonner, rassembler, construire (Koordinieren, sammeln, aufbauen) und soll symbolisch den Anspruch verkörpern, selbst die Kontinuität der früheren Basisaktivitäten der CFDT darzustellen, denn die Formel Coordonner, rassembler, construire war bereits zuvor als Slogan der CFDT im Gesundheitswesen des Großraums Paris benutzt worden.

In den ersten Monaten des Jahres 1989 konsolidieren sich so die ersten ",harten Kerne", aus denen die spätere gewerkschaftliche Strömung rund um die SUD heranwachsen wird. Alsbald sind die GründerInnen vom eigenen Erfolg überrascht. Schon im März 1989 finden die Personalratswahlen bei den PTT statt. Aus dem Stand heraus erhält die neue Organisation SUD-PTT im landesweiten Durchschnitt 5 Prozent der Stimmen. Doch vor allem erhält SUDPTT in der Ile-de-France, der Region von Paris und seines Umlands, auf Anhieb glatte 15 Prozent der Stimmen. Der fulminante Erfolg wird nur den Anfang einer dauerhaften Verankerung in den, künftig getrennten, Unternehmen La Poste und France Télécom bilden.

Vor dem Hintergrund der Wandlungsprozesse dieser beiden Unternehmen im zurückliegenden Jahrzehnt - die Einführung von Managementmethoden wie in der Privatindustrie, der Übergang zu Rentabilitätskriterien bei der Erfüllung vieler öffentlicher Dienstleistungen, die Vorbereitung auf die Privatisierung, die bei France Télécom 1997 erfolgte - konnte SUD-PTT eine bis heute nicht abgerissene Kette von Erfolgen realisieren. Dabei tritt SUD den neoliberalen Umstrukturierungsprozessen mit einer fundamentalen Kritik gegenüber, versteht jedoch zugleich pragmatisch am konkreten Erleben der Beschäftigten anzuknüpfen. Bei den Personalrats- und Betriebsratswahlen bei France Télécom erhielt SUD im Jahr 2000 27,6 und 28 Prozent der Stimmen und liegt damit gleichauf mit der CGT, mit welcher sie sich den ersten Platz teilt. Bei der französischen Post wurde SUD-PTT bei den Personalratswahlen im gleichen Jahr 2000 zur zweitstärksten Gewerkschaft.

In den Krankenhäusern mit ihren anderthalb Millionen Beschäftigten dauerte es für $\mathrm{CRC}$ bis zu einem ähnlichen Erfolg etwas länger, zumal sich Mitte der 90 er Jahre spontan neue Gewerkschaftsgruppen unter dem Namen SUD herausbildeten. Doch der Konflikt konnte gelöst werden, verschiedene Gruppen fusionierten miteinander, und CRC heißt seit 1999 jetzt ihrerseits SUD Santé 
Sociaux (SUD im Gesundheits- und Sozialwesen). Im Großraum Paris erhieit die Gewerkschaft anlässlich der Personalratswahlen 1999 über 16 Prozent der Stimmen. CRC bzw. SUD führt mitunter Aktionen „im Stil vom Mai $1968^{65}$ durch, etwa gelegentliches Einsperren von Direktoren oder Managern. Durch die Mobilisierung bestimmter unterprivilegierter Kategorien von Angestellten - etwa des Küchenpersonals oder der schlecht integrierten Überseefranzosen kann die Gewerkschaft immer wieder Mobilisierungserfolge verzeichnen (Labbé 2001, 62/63).

Die zweite größere Welle von SUD-Gründungen erfolgte in unmittelbarem Zusammenhang mit der breiten Streikbewegung in den öffentlichen Diensten im November und Dezember 1995. Der Ausstand richtete sich unter der konservativen Regierung von Alain Juppé gegen die Demontage der öffentlichen Dienste (bei der Bahngesellschaft SNCF etwa war die Stillegung von 6.000 Streckenkilometern auf einen Schlag wegen „Nichtrentabilität“ geplant), gegen die Angriffe auf die öffentlichen Rentensysteme sowie das Sozialversicherungssystem. Die Führung der CFDT unter ihrer damaligen Generalsekretärin Nicole Notat unterstützte in zentralen Fragen die Regierung Juppé, und verurteilte die breite soziale Bewegung. Dagegen waren rund ein Drittel der CFDTStrukturen auf Ebene der Branchen und Regionen am Ausstand beteiligt.

Durch ihre aktive Teilnahme an der Streikbewegung war einerseits den SUDGewerkschaften und ihrem Dachverband, der Groupe des Dix (G10), zum ersten Mal eine breite Aufmerksamkeit gesichert. Selten sprach man in der Presse bis dahin so viel von den „neuen Gewerkschaften“ (vgl. etwa Le Monde vom 1.12.1995, 6.2.1996 oder 14.4.1996.) Andererseits brachen aber auch die Widersprüche in verschiedenen Sektionen der CFDT in scharfer Form auf. Im Januar und Februar 1996 kam es so binnen weniger Wochen zur sukzessiven Abspaltung mehrerer Gewerkschaftssektionen bei der Bahngesellschaft SNCF von der Transport-Sektion der CFDT.

Die Ausgetretenen gründeten als neue Gewerkschaft im Januar 1996 SUD Cheminots (SUD Eisenbahner), die sich anlässlich ihres ersten Kongresses im März 1997 in SUD Rail (SUD Schienenverkehr) umbenannte - um zu verdeutlichen, dass sie tatsächlich alle bei der SNCF beschäftigten Berufsgruppen einschließlich etwa des Kantinen- oder Reinigungspersonals gewerkschaftlich zu organisieren wünscht. Später konnte SUD Rail auch ausgetretene kritische CGT-Mitglieder hinzugewinnen. Bei den ersten Betriebsratswahlen kurz nach ihrer Gründung, im März 1996, erhielt die Gewerkschaft im nationalen Durchschnitt 4,4 Prozent der Stimmen, obwohl sie in nur 7 von insgesamt 23 SNCF-Großregionen antrat. Im März 2000 erhielt SUD Rail dann 12,73 Prozent der Stimmen.

Die dritte SUD-Gründungswelle resultiert aus der Anwendung der Loi Aubry 1 und Loi Aubry 2, den 1998 bzw. 2000 in Kraft getretenen Gesetzen der Jospin-Regierung zur Arbeitszeitpolitik. Die beiden Gesetzestexte stecken den
Rahmen für den Übergang von der 39-Stunden-Woche zu einer Regelarbeitszeit von 35 Stunden wöchentlich oder 1600 Stunden im Jahr ab. Die konkreten Modalitäten der Arbeitszeitreform sind freilich unter die Bedingung des Abschlusses einzelbetrieblicher Ablommen gestellt; diese werden für die Arbeitgeber attraktiv gemacht, indem bei Abschluss einer entsprechenden Betriebsvereinbarung staatliche Subventionen (in Form des Erlasses von Arbeitgeber-Beiträgen zur Sozialversicherung) in Aussicht gestellt werden. Auf diesem Wege trägt die Reform zu einer „Balkanisierung“ des Arbeitsrechts bei, da die Bedingungen der Arbeitszeitpolitik je nach Größe oder gewerkschaftlicher Tradition des Betriebs völlig unterschiedlich ausfallen könne. Ferner beinhaltet die Reform (durch den Zwang zum Konsens zwischen "Sozialpartnern" auf der betrieblichen Mikroebene) die Notwendigkeit, in vielen Fällen „Gegenleistungen" der abhängig Beschäftigten zu akzeptieren. Diese bestehen in der Regel in der Hinnahme verkürzter, aber flexibler Arbeitszeiten, die je nach Bedarf der Produktion oder der Dienstleistung variieren können.

Die CFDT hatte sich die Unterzeichnung der größtmöglichen Anzahl sozialpartnerschaftlicher Abkommen in diesem Rahmen zum Ziel gesetzt. Doch in vielen Fällen führte dies zu erheblichen Spannungen mit der Mitgliederbasis. Einer der symbolträchtigsten Konflikte bricht beim Reifenhersteller Michelin in Clermont-Ferrand aus. Ein im Januar 2001 vorgestellter Abkommens-Entwurf der Betriebsleitung sieht unter anderem die Ausdehnung der Produktionszeiten auf den (bis dahin in der Regel arbeitsfreien) Samstag vor, mit der Verpflichtung zu regelmäßiger Samstagsarbeit in der Produktion als Gegenleistung für die Arbeitszeitverkürzung. Die Führung der CFDT-Branchensektion setzt die Unterschrift unter das Abkommen durch, während 7 von 10 Sektionen bei Michelin sich strikt dagegen aussprechen. In Folge der Unterzeichnung des Abkommens wird eine Urabstimmung der Beschäftigten der Automobilfirma organisiert. In der Abstimmung am 29. März 2001 wird die Kollektivvereinbarung mit knapp 60 Prozent der Stimmen angenommen. Allerdings kam die Mehrheit aufgrund des Votums der Ingenieure, Techniker und höheren Angestellten zusammen. Dagegen lehnen die hauptsächlich betroffenen Berufsgruppen - Produktionsarbeiter und einfache Angestellte - das Abkommen mehrheitlich ab. Die CFDT bei Michelin zerbricht aufgrund des Konflikts, am Stammsitz des Werks in Clermont-Ferrand treten zwei Drittel ihrer Mitglieder aus und begründen SUD Michelin. Bei den Betriebsratswahlen am 31. Mai 2001 stürzt die CFDT (im Durchschnittsergebnis aller Berufsgruppen) von zuvor 73 auf 49 Prozent der Stimmen ab. Dagegen erhält SUD Michelin auf Anhieb 37 Prozent der Stimmen (Le Monde vom 24.1.2001, 31.03.2001 und 02.06.2001).

Eine erste breitere Verankerung in der Privatindustrie ergibt sich ferner aus dem Übertritt des bisherigen branchenübergreifenden Regionalverbands der CFDT in der Basse-Normandie (die Region um Caen). Der bisher innerhalb des Dachverbands linksoppositionelle Regionalverband war im Mai 1999 „ge- 
kippt" worden. Daraufhin traten zahlreiche Mitglieder und Einzelgewerkschaften aus und begründeten SUD-Gewerkschaften. So entsteht 1999 SUD Industrie, die in Betrieben der Region wie dem (krisengeschüttelten) Küchengerätehersteller Moulinex sowie dem Nutzfahrzeuge-Hersteller RVI (Renault Véhicules Industriels) - der inzwischen von Volvo gekauft wurde - verankert ist. Bei RVI im normannischen Caen erhielt SUD Industrie bei den Betriebsratswahlen im Jahr 2000 eine Mehrheit von 57 Prozent der Stimmen.

\section{Einfluss und Funktionsweise der SUD-Gewerkschaften}

Heute ist SUD landesweit vor allem in öffentlichen Diensten wie bei der Eisenbahn, Post und Telekom, beim öffentlichen Energieversorger und Privatisierungskandidaten EDF - dort hatte SUD Energie im März 2002 einen ersten Wahlerfolg mit 23,9 Prozent im Personalrat der Forschungs- und Entwicklungs-Abteilung. In der Privatindustrie und im Dienstleistungssektor beginnt SUD sich erste Ausgangsbasen für eine breitflächigere Entwicklung zu schaffen. Dabei, bestehen Unterschiede in der Entwicklungslogik: Im privaten Sektor ist es einfacher, örtlich Fuß zu fassen und gute Wahlergebnisse zu erhalten, wenn man über bekannte Mitglieder verfügt. Allerdings ist es in der Folgezeit schwieriger, sich auf der nationalen Ebene auszudehnen, wie in den öffentlichen Diensten, die bereits als nationale Einheiten strukturiert sind. In der Anfangsphase kamen die GründerInnen von SUD-Gewerkschaften oft aus den Reihen der CFDT, nachdem in den Neunzigern deren innere Widersprüche aufgebrochen waren. Besonders seit dem Ende des Jahrzehnts waren aber auch zunehmende Übertritte von Seiten der CGT zu verzeichnen, nachdem - mit dem Ende der von „oben“ her existierenden realsozialistischen Prägung der CGT, und ihrer früher dominierenden KP-Nähe - in dieser eine politische Sinnkrise bzw. ein Verlust klarer gesellschaftlicher Orientierungsmuster ausgebrochen war. Die zunehmend verselbständigte Existenz eines Apparats ohne deutlich hervortretende, handlungsleitende "Richtung" motivierte viele Mitglieder zur Suche nach einer anderen kämpferischen Kraft, die nicht mit der realsozialistischen Hypothek belastet war. Ferner ist in den letzten Jahren auch ein wachsender Zulauf von Mitgliedern ohne Vorlauf in anderen gewerkschaftlichen Organisationen, zu SUD zu verzeichnen.

Die SUD kandidierten im Dezember 1997 und im Dezember 2002 bei den "Sozialwahlen". Dabei werden die als Prud'hommes bezeichneten, paritätisch mit Vertretern der Lohabhängigen und der Arbeitgeber besetzten Arbeitsgerichte gewählt. 17 Millionen Beschäftigte im privaten Wirtschaftssektor sind stimmberechtigt. 1997 konnten die SUD-Gewerkschaften bzw. ihre lockere Koordinierungsstruktur, die Groupe des Dix, landesweit nur 45 Listen aufstellen. Diese erhielten dort, wo sie antraten, im Schnitt 3,4\%, was einem landesweiten Ergebnis von 0,31\% entspricht. 2002 konnten die SUD bzw. ihr um- gewandelter Dachverband Solidaires eine stärkere Präsenz ausüben und mit 176 Listen antreten. Damit standen sie für rund ein Drittel der Wahlberechtigten auf dem Stimmzettel. In drei Vierteln der Fälle konnte das Wahlziel von 5\% der Stimmen (ab dieser Schwelle haben die Kandidaten ein Recht auf Wahlkampfkosten-Rückerstattung) erreicht werden; auf nationaler Ebene ergab das ein Ergebnis von 1,5\% im landesweiten Durchschnitt.

Fast alle SUD-Gewerkschaften gehören einem Dachverband an, der 1981 unter dem Namen Groupe des Dix gegründet wurde. Damals - SUD existierte noch nicht - ging es vor allem darum, jene "autonomen " (d.h. keinem Dachverband angehörenden) Einzel- und Branchengewerkschaften zusammenzuschließen, die meist seit der Spaltung zwischen den Dachverbänden CGT und FO in den Jahren 1947/48 außerhalb nationaler Bundesstrukturen verblieben waren. Das anfängliche Koordinierungstreffen von 10 teilweise kämpferischen (wie der traditionsreichen Gewerkschaft der Finanzbediensteten SNUI), teilweise eher konservativ-unpolitischen Organisationen blieb in den ersten Jahren ohne tiefere Perspektive oder verbindliche Ziele. Auf Einladung des SNUI, der 1989 selbst einen kämpferischen mehrmonatigen Streik in den Finanzämtern geführt hatte, trat die frisch gegründete SUD-PTT dieser Koordinierungsstruktur als „Beobachter" bei. 1992 erwarb SUD-PTT die Vollmitgliedschaft in der "Gruppe der Zehn". In den darauf folgenden Jahren traten die sich neu gründenden SUD-Gewerkschaften überwiegend diesem Verband bei, der anlässlich der Kongresse von 1998 und 2001 zum Dachverband Union syndicale - Solidaires umgebaut wurde. Gleichzeitig verließen einige der konservativsten Organisationen die Koordinierungsstruktur, um dem „Verband der unabhängigen Gewerkschaften" UNSA - einem eher unpolitischen, aber der CFDT nahe stehenden Koordinierungsverband - beizutreten.

Die Union Syndicale - Solidaires weist heute 32 nationale Mitgliedsorganisationen auf, die wiederum rund 80.000 Einzelmitglieder umfassen. ${ }^{4}$ Ungefähr die Hälfte der Unions-Mitglieder gehört SUD-Gewerkschaften an, die andere Hälfte aus anderen Traditionen stammenden Organisationen, von denen sich aber ein Teil in eine ähnliche Richtung entwickelt. Die größten Mitgliederorganisationen sind der SNUI mit rund 20.000 Mitgliedern und SUD-PTT mit rund 16.000 Organisierten.

Als Funktionsprinzipien wurden sowohl innerhalb der einzelnen SUDGewerkschaften (in der Regel), als auch im Dachverband Union syndicale -

4 In Frankreich ist der gewerkschaftliche Organisationsgrad insgesamt aufgrund verschiedener Faltoren, darunter die antigewerkschaftliche Repression vor allem in kleineren und mittleren Betrieben und der Anstieg der Erwerbslosigkeit sowie voraus gegangene Niederlagen der Arbeiterbewegung, stark gesunken. Er beträgt derzeit nur noch 8 bis 10 Prozent der Beschäftigten. Damit sind heute insgesamt zwischen 1,5 und 2 Millionen Beschäftigte Gewerkschaftsmitglieder. Die Möglichkeit zur von Gewerkschaftsfïhtungen unabhängigen Ausübung des Streikrechts und andere Faktoren müsen jedoch den Findruck tergrund herrsche soziale Friedhofsruhe. 
Solidaires basisdemokratische Grundregeln festgelegt. So gab SUD-PTT sich im Jahr ihrer Gründung, 1989, eine "Charta zur Selbstdefinition". In ihr wurden die strikte Begrenzung der Zahl von Hauptamtlichen, die Rotation in gewerkschaftlichen Ämtern (die Obergrenze wurde zunächst auf zwei aufeinander folgende Mandate von drei Jahren festgelegt), ein Mindestanteil an Frauen von 35 Prozent - das entspricht ihrem Anteil an der Mitgliedschaft - und die gleichmäßige Verteilung von "politischen“ und "technischen" Aufgaben auf alle Funktionsträgern festgeschrieben. Dadurch sollte die Herausbildung einer hauptamtlichen Gewerkschaftsbürokratie, wie in den großen Dachverbänden, verhindert werden. In 13 Jahren Existenz scheint SUD-PTT ihre selbst gesteckten Prinzipien größtenteils eingehalten haben. Aufgrund des starken Zuwachses an Neumitgliedern, oft ohne vorherige gewerkschaftliche Erfahrung, sind einige Prinzipien in jüngerer Zeit etwas gelockert worden. So sind nunmehr drei statt zuvor zwei (jeweils dreijährige) Amtszeiten gestattet, um den „Übergang langjähriger Erfahrung “ auf die jüngeren Mitglieder zu gewährleisten. Allerdings hat die Rotation im Sprecheramt von SUD-PTT funktioniert: 1999 wurde das Gründungsmitglied Annick Coupé durch Joëlle Charuel abgelöst, diese wiederum im Jahr 2002 durch René Ollier. Im Dachverband Solidaires, der nach wie vor keinen Gewerkschaftsbund im klassischen Sinne darstellt, wurden strikte demokratische Prinzipen festgeschrieben. So lautete die Regel ursprünglich, dass Entscheidungen nur im Konsens aller vertretenen Basisorganisationen gefällt werden können. Aufgrund der Notwendigkeit, immer häufiger Entscheidungen zu treffen, wurde die Regel abgeschwächt. Einerseits ist die mehrheitliche Zustimmung aller Mitgliedsorganisationen erforderlich, die jeweils - unabhängig von ihrer Mitgliederzahl - eine Stimme aufweisen. Andererseits verfügt jede Einzelgewerkschaft über ein Rückzugsrecht (das bedeutet, dass sie sich an einer ihrer Ideen widersprechenden Aktion nicht beteiligen muss) sowie ein Vetorecht: Widerspricht eine Aktion tragenden Grundprinzipien einer Mitgliedsgewerkschaft, so kann sie diese durch ihr Veto verhindern.

Die Anhänger und Sympathisanten der SUD- bzw. Solidaires-Gewerkschaften weisen einige politische Besonderheiten auf. So ergab die Auswertung einer Umfrage unter Gewerkschafts-Sympathisanten im April 2002, dass die SUDSympathisanten anlässlich der Präsidentschaftswahl unter allen Gewerkschaftsanhängern (mit weitem Abstand) am wenigsten für die rechtsextremen Kandidaten Jean-Marie Le Pen und Bruno Mégret gestimmt hatten. Insgesamt erhielt die extreme Rechte bei denen, die sich als SUD-Sympathisanten ausgeben, nur 3\% (im Vergleich zu durschnittlich 20\% der Franzosen, 12\% unter den CFDT-Parteigängern und $25 \%$ unter den Anhängern der christlichen CFTC). Zugleich stimmen 40\% unter ihnen für die radikale Linke, der größte Teil unter ihnen (27\%) für den marxistisch-undogmatisch auftretenden Kandidaten der Ligue Communiste Révolutionnaire (LCR), Olivier Besancenot - der 27 Jahre junge Kandidat und Postbeschäftigte ist selbst Mitglied von SUD-PTT. Unter den Mitgliedern finden sich von Anfang an sowohl Vertreter anarchistischer und libertär-kommunistischer Strömungen wie auch der beiden trotzkistischen Parteien LCR und LO, aber mittlerweile auch Sozialdemokraten und Grüne. Unter den Neumitgliedern befinden sich inzwischen freilich auch viele ohne politische Vorgeschichte.

Über die soziale Zusammensetzung der Mitglieder- und Sympathisantenbasis dürfte soviel feststehen: Erstens hat SUD eine bedeutende Verankerung unter den Beschäftigten der (bisherigen) öffentlichen Dienste. Dies steht in Zusammenhang mit dem fundamentalen Rollenwandel dieser Dienste im Kontext von neoliberaler Politik, Rentabilitätsdruck und Privatisierungsoffensiven und der damit einhergehende Infragestellung der beruflichen Rolle der Beschäftigten. In bestimmten öffentlichen Diensten wie bei der Post begannen in den 70er Jahren oftmals sozial schlechter integrierte Gruppen in überdurchschnittlichem Maße zu arbeiten, etwa Überseefranzosen (von den Antillen oder La Réunion) oder auch manche „Provinzler". Der öffentliche Sektor versprach bei schlechterer Bezahlung - zumindest Arbeitsplatzsicherheit und erleichterte es in der „Metropole" Fuß zu fassen, bei gleichzeitiger Aussicht auf eine spätere „Versetzung“ in Richtung Heimat. Zugleich zog der Postdienst damals oft junge Linke und „postmaterialistisch"-alternativ eingestellte Personen an, da zwar die Bezahlung im Durchschnitt deutlich geringer, aber die Arbeitshetze erheblich schwächer war als in der privaten Wirtschaft (in der damals noch leicht Arbeitsplätze zu finden waren). Zudem wirkte die hohe Konzentration von Arbeitern, bei gleichzeitig ausgeprägter gewerkschaftlicher Präsenz und damals - noch - relativ lockeren Arbeitsbedingungen, attraktiv auf diese Gruppe. Diese Beschäftigtenstruktur, jedenfalls der „Alteingesessenen“, liefert eine Teilerklärung für die erfolgreiche Etablierung von SUD im PTT-Sektor.

Hinzu kommt, zweitens, in jüngerer Zeit eine erhöhte Attraktivität von SUD für die Kinder des „Bildungsbooms ${ }^{66}$, also der verlängerten Ausbildungs- und Studienzeiten, die heute - oft weit unter ihrer Qualifikation - namentlich im Dienstleistungssektor beschäftigt sind. Dieses „akademische Proletariat" legt, neben Anforderungen an Lohngerechtigkeit besonderen Wert auf „postmaterielle" Faktoren wie halbwegs respektvolle Behandlung, minimale Möglichkeiten individueller Entfaltung auch im Arbeitsleben u.ä. Es bildet eine wichtige Basis für die Entwicklung von SUD-Gewerkschaften etwa in den Forschungsund Entwicklungsabteilungen von Großunternehmen (wie jener des Energieversorgers EDF, oder bei den Entwicklungsingenieuren des Automobilherstellers Renault), oder auch beim Dienstleistungsanbieter FNAC. In der, auf Kulturartikel sowie Informatik spezialisierten Kaufhauskette hat SUD eine örtlich starke Verankerung in mehreren Pariser Filialen und spielte im Jahr 2002 eine wichtige Rolle bei der Streikbewegung gegen bestehende Lohn-Ungleichheiten zwischen verschiedenen Pariser FNAC-Niederlassungen. 
Ein prägendes Merkmal der SUD-Gewerkschaften ist ihre Weigerung, ihre Aktivitäten auf den Betrieb und rein quantitative Forderungen zu beschränken. Schon in ihrem Gründungsjahr 1989 nahm SUD-PTT an der damaligen Kampagne für einen Schuldenerlass für „Dritte Welt"-Länder teil und engagiert sich für das Wahlrecht für Immigranten. 1990/91 engagierte sich die noch kleine Gewerkschaft in der Bewegung gegen den Golfkrieg. Seitdem hat das gesellschaftliche Engagement der SUD-Basisgewerkschafter vielfältige Formen angenommen. Die „Pionierorganisation" SUD-PTT etwa ist 1994 Gründungsmitglied des Bündnisses AC! (Agir ensemble contre le chômage, Gemeinsam handeln gegen Arbeitslosigkeit), das die Proteste der Erwerbslosen zu strukturieren versucht. 1998 ist SUD-PTT eines der kollektiven Gründungsmitglieder des globalisierungskritischen Netzwerks ATTAC in Frankreich. SUD-PTT ist an der Seite der Sans papiers, der um ihre Rechte kämpfenden ,illegalen “ Immigranten, ebenso engagiert wie in internationalistischen Kampagnen. Im Dezember 2000 organisierten SUD-Gewerkschafter beim Versandbuchhandel Amazon.fr den wahrscheinlich ersten Solidaritätsstreik (seit Jahrzehnten) in Frankreich für US-amerikanische Arbeiter. Einige, aber nicht alle SUD-Gewerkschaften haben kritische Positionen zur Atomenergie bezogen, so SUD Rail, die sich über die Beschäftigung mit nuklearen Gefahrgütertransporten per Eisenbahn in das Thema eingearbeitet hatte und inzwischen an koordinierten Aktionen mit dem Anti-Atomkraft-Netzwerk Sortir du nucléaire teilnimmt.

Annick Coupé hebt es in der Selbstdarstellung von SUD/Solidaires (Coupé 2002) an zahlreichen Stellen hervor: Aufgabe einer gewerkschaftlichen Solidarität sei es, den Blick über den Horizont der eng umrissenen Eigeninteressen im Betrieb hinaus zu lenken, und alle gesellschaftlichen Konsequenzen ,des Neoliberalismus als derzeitiger Form des Kapitalismus" zusammen zu denken. Gewerkschaftliche Aktivität sei in dieser Form auch ein Mittel, „um die Welt, in der wir leben, besser verstehen zu lernen“. Im Unterschied zu traditionellen Gewerkschafts-Dachverbänden, die sich in der Vergangenheit auf linke Ideologien bezogen, wird dieser unterschiedliche gesellschaftliche Aspekte umspannende Diskurs aber nicht von oben aufgepfropft. Die Positionen zu gesamtgesellschaftlichen Fragen sollen durch permanente, immer wieder begonnene Diskussionsprozesse zwischen den einzelnen Organisationen und mit ihren Mitgliedern erarbeitet und vertieft werden.

Ein letztes Strukturmerkmal der SUD-Gewerkschaften ist ihre Beziehung zur (nicht gewerkschaftlich organisierten) Basis, also zu den abhängig Beschäftigten. SUD hat sich systematisch Transparenz statt Geheimpolitik auf die Fahnen geschrieben und lehnt Verhandlungen ohne Informationsfluss an die „Basis“ ab. So beklagt sich, in einer den Arbeitgebern nahe stehenden Fachzeitschrift, der Mitarbeiter einer Consulting-Firma: „SUD Informationen unter dem Vorbehalt der Vertraulichkeit anzuvertrauen, ist schlicht irrig, denn alles wird öffentlich gemacht" (Trentesaux, 74). Dort, wo SUD als „repräsentativ" in oben beschriebenen Sinne anerkannt ist, ist die Arbeitgeberseite allerdings rechtlich dazu verpflichtet, ihr die selben Informationen wie anderen gewerkschaftlichen Verhandlungspartnern zukommen zu lassen. SUD-Gewerkschaften publizieren häufig detaillierte Analysen über Kollektivverträge bzw. Entwürfe dafür, die sich in Verhandlung befinden oder abgeschlossen wurden unter genauer Herausarbeitung dessen, was für die Beschäftigten auf dem Spiel steht. Zugleich praktiziert SUD aber keine systematische Verweigerung, was den Abschluss von Betriebsvereinbarungen betrifft, vor allem dort, wo die Beschäftigten - nachdem sie gründlich informiert wurden - dem mehrheitlich positiv gegenüber stehen, oder wo nach einem erfolgreichen Streik Fortschritte festgeschrieben werden sollen. Vor allem aber praktiziert SUD eine Informationspolitik, die eigene Niederlage oder vorherige Fehleinschätzungen nicht verschweigen oder schönreden möchte, etwa nach einem Arbeitskampf, dessen Ergebnisse ungünstig ausfallen. Auch darin liegt ein Unterschied zu manchen „klassischen “ Gewerkschaftsorganisationen.

\section{Fazit und Ausblick}

13 Jahre nach ihrer Gründung innerhalb der damals noch einheitlichen französischen Post und Telekom sind die SUD-Gewerkschaften nicht mehr aus der politisch-sozialen Landschaft Frankreichs wegzudenken. Die damaligen Initiatoren hätten sich das 1988/89 kaum träumen lassen. Zumindest in einigen wirtschaftlichen Sektoren und gesellschaftlichen Bereichen haben die innovativen Praktiken der alternativ-basisdemokratisch ausgerichteten Organisation dauerhafte Erfolge gezeitigt - vor allem in den (bisherigen) öffentlichen Diensten. Aber auch im privaten Wirtschaftssektor beginnt das "Modell ${ }^{66}$ SUD da und dort Fuß zu fassen. Landesweit jedoch bilden SUD-Solidaires noch keinen Verband, der mit den traditionellen Gewerkschaftsbünden - den confédérations - auf quantitativer Ebene gleichziehen könnte. Bestimmt aber tragen sie dazu bei, eine neuartige und vorwärts weisende Antwort auf die auch in Frankreich grassierende - gewerkschaftliche Krise zu geben. Sie zeigen, dass andere gewerkschaftliche Praktiken als jene hoch bürokratisierter, schwerfälliger und (bezogen auf ihre eigene Funktionsweise) strukturkonservativer Organisationen möglich sind. Und dass es zugleich möglich ist, diese neue Form gewerkschaftlicher Aktivität in enger Bindung an die Vielfalt gesellschaftlicher Widerspruchsfelder zu entwickeln. Insofern ist die Krise auch eine Chance.

Die Erfolgsgeschichte von SUD/Solidaires erklärt sich teilweise aus spezifisch französischen, historischen und gesellschaftlichen Faktoren. Da ist die Krise der vormals für die Arbeiterbewegung des Landes (in Teilen) prägenden positiven Bezugs auf den sowjetischen Kommunismus. Da ist vor allem die relati- 
ve Schwäche - verglichen etwa mit der deutschen Situation - der (miteinander rivalisierenden) traditionellen, hoch bürokratisierten Gewerkschaftsapparate. Diese quantitative Schwäche, verbunden mit einer fest in der französischen Geschichte verwurzelten Kampfbereitschaft für soziale und demokratische Ziele innerhalb breiter Teile der Gesellschaft und der französischen („nicht organischen") Konzeption des Streikrechts, erleichtert die Autonomie „der Klasse" - und neuer Akteure in ihr - gegenüber jenen Apparaten, die sich längst, wie im Fall der CFDT, zum Disziplinierungsinstrument im Sinne herrschender Ordnungspolitik verwandelt haben. Es ist zu wünschen, dass diese neuen Akteure im Bündnis mit anderen Gruppen - etwa Teilen der CGT, die sich in Frage zu stellen vermögen - Antworten auf die Krise der französischen Gewerkschaftsbewegung entwickeln können.

\section{Quellen}

1. Interview des Autors mit

- Guy Freyche, Gründungsmitglied von SUD-PTT (Dezember 1995 und Dezember 2002)

- Annick Coupé, Sprecherin des Dachverbands Union syndicale - Solidaires (Dezember 2002)

- Yann Cochin und Pierre Masnières, Führungsmitglieder von SUD Energie (Dezember 2002)

- Dominique Malvaud, Führungsmitglied von SUD Rail (November 2002)

- Alain Martinez, Mitgründer von SUD Renault im Forschungszentrum des Automobilkonzerns in Guyancourt (Dezember 2002)

2. Literatur

Aparicio, Jean-Claude/Pernet, Michel/Torquéo, Daniel (1999): La CFDT au péril du libéral syndicalisme, Paris.

Coupé, Annick (2002): Qu'est-ce que SUD Solidaires?, in L'information citoyenne, Paris.

Cretticz, Xavier / Sommier, Isabelle (Hg. 2002): La France rebelle. Tous les foyers, mouvements Cretticz, Xavier / Sommier, Isabell
acteurs de la contestation, Paris.

acteurs de la contestation, Paris.
Hamon, Patrick/ Rotman, Patrick (1982, erweiterte Neuauflage 1984/2002): La deuxième gauche. Histoire intellectuelle et politique de la CFDT, Paris.

Kesselmann, Mark (Hg.), Le mouvement ouvrier français 1968 - 1982. Crise économique et changement politique, Paris.

Labbé, Daniel/Landier, Hubert (2001): L'entreprise face au nouveau radicalisme syndical, Paris. Les votes au scrutin présidentiel selon la proximité syndicale, in: Liaisons sociales, 30 April 2002.

Mouriaux, René (1984): La C.F.D.T.: de l'union des forces populaires à la réussite du changement social, in: Kesselmann, Mark ( $\mathrm{Hg}$.)

Ross, George: La C.G.T: crise économique et changement politique, in: Kesselmann, Mark (Hg.) Trentesaux, Jacques : Social : La montée des extrémistes, in: Enjeux - Le mensuel de l'économie,
Novembre 2002.

\section{Raul Zelik}

\section{Gewerkschaftssterben in Kolumbien Über den Zusammenhang von neoliberaler Deregulie- rung, staatlicher Politik und Paramilitarismus}

Wird über Gewerkschaftspolitik diskutiert, ist schnell von 'Krisen' die Rede. Aber woran macht sich die Krise von Gewerkschaften eigentlich fest? An niedrigen Mitgliederzahlen oder der Kooptation von Apparaten im Rahmen der Sozialpartnerschaft? An der Bewegungslosigkeit von Großgewerkschaften oder an der Existenz kleinerer, konkurrierender Gewerkschaften, die uneinheitlich vorgehen, aber dafur gesellschaftspolitische Prozesse in Gang setzen können? Von den meisten DGB-Funktionären dürfte die 'Krise' wohl vor allem als Verlust von Verhandlungsmacht in den Zeiten neoliberaler Deregulierung wahrgenommen werden. Für Gewerkschaftslinke wie die Aktivisten der Bayer-Liste in Wuppertal, die Anfang der 80er Jahre auf schmutzigste Weise von der IG Chemie-Führung hintergangen wurden, hat die Krise der deutschen Gewerkschaften hingegen zu einem ganz anderen Zeitpunkt begonnen. In diesem Text verstehe ich 'Krise' vor allem als existenzielles Problem. Sie beschreibt die Verdrängung gewerkschaftlicher Organisation im Allgemeinen und die Eliminierung gewerkschaftslinker/regierungsoppositioneller Positionen im Besonderen, denn in Kolumbien - man muss es so dramatisch ausdrücken - findet seit Ende der 80er Jahre ein regelrechter Vernichtungsfeldzug gegen Gewerkschaften statt. Verantwortlich dafür sind, wie gezeigt werden soll, Reformen im Arbeitsrecht, staatliche Kriminalisierung und eine Mordkampagne, die ihresgleichen sucht. Allein 2001 wurden nach Angaben der International Confederation of Free Trade Unions (ICFTU) (2002) mehr als 200 Gewerkschafter in dem südamerikanischen Land ermordet (vgl. Pérez/Colectivo de Abogados 2002). Das US State Department (2002), gewerkschaftsfreundlicher Übertreibungen sicherlich unverdächtig, geht davon aus, dass in Kolumbien seit 1991 insgesamt 1600 Gewerkschafter getötet und 60 Prozent der Morde an Gewerkschaftern weltweit in dem südamerikanischen Land verübt wurden. Dabei bilden Mordfälle nur die Speerspitze der Angriffe. Die jesuitennahe Forschungseinrichtung CINEP hat im Jahr 2000277 Angriffe auf Gewerkschaftsführer und 870 Angriffe auf Gewerkschaftsmitglieder gezählt

PROKLA. Zeitschrift firr kritische Sozialwissenschaft, Heft 130, 33. Jg., 2003, Nr. 1, 51-75 